\begin{tabular}{|c|c|}
\hline Title & Human intestinal spirochetosis is significantly associated with sessile serrated adenomas/polyps \\
\hline Author(s) & $\begin{array}{l}\text { Omori, Saori; Mabe, Katsuhiro; Hatanaka, Kanako; Ono, Masay oshi; Matsumoto, Mio; T akahashi, Masakazu; Y oshida, } \\
\text { T akeshi; Ono, Shoko; Shimizu, Y uichi; Sugai, Nozomi; Suzuki, A kira; Katsuki, Shinichi; Fujii, T akahiro; Kato, } \\
\text { M ototsugu; A saka, Masahiro; Sakamoto, Naoya }\end{array}$ \\
\hline Citation & $\begin{array}{l}\text { Pathology, Research and Practice, } 210(7), 440-443 \\
\text { https://doi .org/10.1016/.prp.2014.03.007 }\end{array}$ \\
\hline Issue Date & 201407 \\
\hline Doc URL & http:/hdl.handle.net/2115/56700 \\
\hline Type & article (author version) \\
\hline File Information & Pathol ResPract_210(7)_440-443.pdf \\
\hline
\end{tabular}

Instructions for use 
Title:

\section{Human Intestinal Spirochetosis Is Significantly Associated with Sessile Serrated}

\section{Adenomas/Polyps}

Saori Omori, ${ }^{1,2 \S}$ Katsuhiro Mabe, ${ }^{1 * \S}$ Kanako Hatanaka, ${ }^{3}$ Masayoshi Ono, ${ }^{1,2}$ Mio Matsumoto, ${ }^{1,2}$ Masakazu Takahashi, ${ }^{1,2}$ Takeshi Yoshida, ${ }^{2}$ Shoko Ono, ${ }^{1}$ Yuichi Shimizu, ${ }^{2}$ Nozomi Sugai, ${ }^{4}$ Akira Suzuki, ${ }^{5}$ Shinichi Katsuki, ${ }^{6}$ Takahiro Fujii, ${ }^{7}$ Mototsugu Kato, ${ }^{1}$ Masahiro Asaka, ${ }^{8}$ Naoya Sakamoto $^{2}$

${ }^{1}$ Division of Endoscopy, Hokkaido University Hospital, Sapporo, Hokkaido, Japan

${ }^{2}$ Department of Gastroenterology, Hokkaido University Graduate School of Medicine, Sapporo,

Hokkaido, Japan

${ }^{3}$ Division of Pathology, Hokkaido University Hospital, Sapporo, Hokkaido, Japan

${ }^{4}$ Division of Gastroenterology, KKR Sapporo Medical Center, Sapporo, Hokkaido, Japan

${ }^{5}$ Division of Pathology, KKR Sapporo Medical Center, Sapporo, Hokkaido, Japan

${ }^{6}$ Center of Gastroenterology, Otaru Ekisaikai Hospital, Otaru, Hokkaido, Japan

${ }^{7}$ Takahiro Fujii Clinic, Tokyo, Japan 
${ }^{8}$ Department of Cancer Preventive Medicine, Hokkaido University Graduate School of Medicine, Sapporo, Hokkaido, Japan

Running title: Association between human HIS and SSA/Ps

$\S$ S.O. and K.M. contributed equally to this work

*Corresponding Author:

Division of Endoscopy, Hokkaido University Hospital

Address: North 14, West 5, Kita-ku, Sapporo, Hokkaido 060-8648, Japan

Phone: +81 117161161 (ex. 5920)

Fax: +81 117067867

E-mail: kmabe@med.hokudai.ac.jp 


\section{Abstract}

Aims: It remains unclear whether or not human intestinal spirochetosis (HIS) has any associated symptoms or lesions. In this study, we assessed the prevalence of HIS in sessile serrated adenomas/polyps (SSA/Ps) and their possible association.

Methods: Following identification of early cecal cancer with SSA/P accompanied by a colonization of HIS, we went on to conduct a retrospective, case-control study, using endoscopically resected SSA/P specimens to examine the frequency of HIS infection in SSA/Ps. Results: Nineteen SSA/P cases and 172 controls were obtained. The rate of HIS infection was significantly higher at $52.6 \%(10 / 19)$ in the SSA/P cases compared to the controls at $8.1 \%$ $(14 / 172)$.

Conclusions: Our SSA/P series were associated with a remarkably higher rate of HIS than controls or than previously reported. This is the first report to provide evidence for potential association between HIS and SSA/Ps.

\section{Introduction}

Among the heterogeneous group of colonic serrated lesions, sessile serrated adenomas/polyps (SSA/Ps) are reported to be associated with malignant alternations, despite their resemblance to hyperplastic polyps (HPs) in gross morphology, through the serrated pathway involving the 
mutation of the BRAF gene and DNA methylation [1], although the causal factors responsible for such mutation and methylation remain yet to be elucidated. On the other hand, while spirochetes are well-known causative pathogens of watery diarrhea in veterinary medicine [2], there are conflicting reports $[3,4]$ as to whether human intestinal spirochetosis (HIS), which is mostly caused by Brachyspira aalborgi (B. aalborgi) or B. pilosicoli, is associated with symptoms of chronic diarrhea or irritable bowel syndrome. It is also reported that HIS may be responsible for colonic adenomas or hyperplastic polyps (HPs) [5], while its association with neoplasms remains largely unclear [3].

Following identification of a HIS-positive patient in whom both SSA/P and synchronous adenocarcinoma were present (Figure 1), we conducted a retrospective, case-control study, by using endoscopically resected SSA/P specimens to examine the prevalence of HIS in SSA/Ps and their association.

\section{Subjects and Methods}

In this case-control study, SSA/P cases consisted of SSA/P lesions endoscopically removed at our hospital and affiliated institutions between January 2008 and December 2011, and controls consisted of colon biopsies endoscopically obtained from non-SSA/P patients at our hospital between July and December 2011. All specimens were fixed in 10\% formaldehyde for at least 
24 hours. Given that spirochetes are reported to attach themselves to the microvilli of the colon

[6], control specimens were excluded if they had been taken from patients with advanced colorectal cancer, as they are thought likely to be associated with minimal endothelial microvilli, thus potentially contributing to a low HIS rate among the control specimens.

For all patients diagnosed with SSA/Ps at the participating institutions, endoscopic images, hematoxylin and eosin (HE)-stained and unstained histologic specimens of the lesions were collected for central review at Hokkaido University. Of these, those confirmed by two independent pathologists to be consistent with the findings characteristic of SSA/Ps [7] were included for analysis. In their diagnosis, attention was focused on whether the lesions were associated with two or more of the following: 1) crypt dilation; 2) irregular branching crypts; or 3) horizontally arranged basal crypts (inverted T- and L-shaped crypts) [8] (Figure 2).

All histologic specimens were examined for presence of HIS by HE staining and immunostaining with anti-Treponema pallidum (TP) antibody which is reported to present a cross reaction with the Brachyspira species (AbD Serotec, UK) [9]. Specimens were judged to be HIS-positive if the colonic brush border was found HIS-positive after immunostaining (Figure 3A) and if the spirochetes were found to be present in the surface or crypt mucus (Figure 3B). 
Statistical analysis

Statistical analyses were performed to compare cases and controls by using Fisher's exact test. All statistical analyses were performed by using SPSS 20 (SAS Institute Inc., Cary, NC, USA). $P$ values of $<0.05$ were considered statistically significant.

\section{Results}

Thirty-five SSA/P cases (19 patients; men, 63.9\%; mean age, 62.3 years) and 348 controls (172 patients; men, 66.9\%; mean age, 54.6 years) were obtained and subjected to central review and analysis (Table 1). All SSA/Ps were shown to be flat, proximally located, and associated with multiple synchronous lesions, including HPs, adenomas and adenocarcinomas. Three of the 10 HIS-positive SSA/P cases were associated with adenocarcinomas, with 2 found in the same sites as the SSA/Ps and 1 found in a different site.

All control specimens were biopsy samples, single or multiple, taken from the colon and rectum of the same control patients, and there was no difference in HIS infection rate between these biopsy sites at $7.3 \%$ in the right colon and at $8.1 \%$ in the left rectum (Figure 4$)(P=$ 0.796). Again, there was no significant difference in HIS infection rate, irrespective of whether single or multiple biopsies were taken from the same control patients (6.3\% and 10.4\%; 6/95 and $8 / 77, P=0.331)$. 
The rate of HIS infection was significantly higher at 52.6\% (10/19) in SSA/P cases than in controls at 8.1\% (14/172) $(P<0.001$; Fisher’s exact test) (Figure 5).

Of the 14 HIS-positive controls, very few were associated with "fringe formations" thus failing to meet the diagnosis of HIS at the initial diagnosis based on HE staining alone. However, a more detailed examination of the controls involving immunostaining revealed that 9 of the 14 controls were associated with "fringe formations" on HE staining.

Of the 10 HIS-positive SSA/P patients, 4 patients were judged to be positive for HIS both on HE staining (i.e., "fringe formation” present) and on immunostaining, while the remaining 6 were judged to be positive for HIS based on immunostaining, while they showed no "fringe formation" on HE staining. Again, spirochetes were found to be present in both the SSA/P lesions and their background mucosa in 4 patients (40\%), in the SSA/P lesions alone in 4 (40\%), and in the background mucosa alone in 2 (20\%). Spirochetes were found to be present on the surface of the mucosa that remained intact.

There was no clear difference between the HIS-positive and -negative SSA/P cases in regard to the degree of lymphocyte infiltration in the background mucosa, amount of mucus on the surface mucosa, or crypt length and breadth (data not shown).

\section{Discussion}


Following identification of a case of SSA/P associated with multiple SSA/P lesions covered by mucus, we suspected and examined the presence of some bacterial infection which may account for the onset of SSA/Ps. Study results demonstrated that SSA/Ps as a potential causal factor for the onset of colon cancer are associated with a higher rate of HIS infection than controls, thus strongly suggesting an association between HIS infection and the onset of SSA/Ps.

In recent years, SSA/Ps have come to be characterized as the $\mathrm{CpG}$ island methylator phenotype-high (CIMP-high) associated with the mutation of the BRAF gene and regarded as precursor lesions of colorectal cancer. However, the causal factors responsible for such gene mutation or methylation remain largely unknown. In this regard, our results suggest that HIS may be implicated in the pathogenesis of SSA/Ps or their malignant alterations, while they fall short of providing definite evidence for their causal relationship.

In gastric cancer in which Helicobacter pylori (H. pylori) infection-associated, aberrant methylation is shown to be implicated [10], eradication of $H$. pylori has been shown to reduce the risk of developing gastric cancer not only in animal studies, but also in a prospective, multicenter, randomized, controlled trial [11].

If HIS is to be implicated in the development of SSA/P and/or its malignant alterations, eradication of spirochetes may have a role in reducing the risk of developing colon cancer in a subset of patients, as with $H$. pylori eradication in gastric cancer. Therefore, further studies are 
warranted to confirm our findings.

In this study, all specimens were examined for presence of HIS by using both HE staining and immunostaining, which detected HIS infection in a greater proportion of control specimens than was possible in an earlier Japanese report [9] and allowed detection of control specimens associated with a "fringe" formation along the colonic brush border, which may not be characterized as such by HE staining alone. However, our study has several limitations. First, the study involved a small sample size and it was designed as a retrospective, case-control study, although it may be difficult to prospectively investigate the association between HIS and SSA/Ps, given that SSA/Ps are less common in incidence than other colonic adenomas. Second, the endoscopically resected specimens and biopsy specimens differed in regard to their size and location, although this may not be too much of an issue, given that the incidence of HIS did not differ between the biopsy sites examined. In this regard, a comparison of another $10 \mathrm{SSA} / \mathrm{P}$ patients and their 10 tubular adenoma controls matched for sex, age, treatment phase, and lesion location/size showed that 9 of 10 (90\%) SSA/P patients were found to be positive for HIS, and 4 of $10(40 \%)$ tubular adenoma controls were found to be positive for HIS (no detailed data shown), suggesting that the HIS infection rate may not vary depending on the specimen size or location. Third, the study confined itself to evaluating HIS based on cytological and histological diagnosis alone. More in-depth analysis with PCR may be required to establish the rate of HIS 
infection and to identify the B. species involved, including B. aalborgi and B. pilosicoli. Again, in-vitro studies involving cell cultures or animal experiments are required to elucidate the association between HIS and SSA/P-related gene mutations and/or methylation, in order to provide further evidence for association between HIS and SSA/Ps.

\section{Conclusions}

Our SSA/P cases were associated with a remarkably higher rate of HIS than controls or than previously reported. Study results suggest that HIS is among the factors responsible for the pathogenesis of SSA/Ps. To the best of our knowledge, this is the first report to provide evidence for potential association between HIS and SSA/Ps. Further studies are warranted to examine HIS for association with SSA/P-related gene mutations and/or methylation.

\section{Take home message}

SSA/P cases were associated with a remarkably higher rate of HIS than controls. Immunostaining using anti-TP antibody is useful for detection of HIS.

\section{Funding None.}

\section{Competing interests None.}


Contributors Study concept and design: KM, MK. Acquisition of data: SM, KM, MO, MM, MT, TY, SO, YS, NS, AS, SK, and TF. Analysis and interpretation of data: SO, KM, KH, MO, and AS. Drafting of the manuscript: SO, KM. Critical revision of the manuscript for important intellectual content: KH, SK, MK, MA, and NS. Administrative, technical or material support: MK, MA, and NS. Study supervision: KM, MK, MA, and NS. All authors had full access to all of the data in the study and take responsibility for the integrity of the data and the accuracy of the data analysis.

\section{References}

1. Leggett B, Whitehall V. Role of the serrated pathway in colorectal cancer pathogenesis. Gastroenterology 2010;138(6):2088-2100.

2. Smith JL. Colonic HIS in animals and humans. J Food Prot 2005;68(7):1525-34.

3. Calderano A, Bommezzadri S, Corrini C, et al. Infective colitis associated with human HIS. J Gastroenterol Hepatol 2007;22(11):1772-9.

4. Anthony NE, Backwell J, Ahrens W, Lovell R, Scobey WW. HIS: an enigmatic disease. Dig Dis Sci 2013;58(1):202-8.

5. Calderano A, Gorrini C, Montecchini S, et al. Intestinal spirochaetosis associated with hyperplastic and adenomatous colonic polyps. Pathol Res Pract 2012;208(3):177-180. 
6. Harland WA, Lee FD. Intestinal spirochaetosis. Br Med J 1967;3(5567):718-719.

7. Higuchi T, Sugihara K, Jass JR. Demographic and pathological characteristics of serrated polyps of colorectum. Histopathology 2005;47(1):32-40.

8. Yao T, Sugai T, Iwashita A, et al. Histopathological characteristics and diagnostic criteria of SSA/P, from Project Resaerch "Potential of Cancerization of Colorectal Serrated Lesions" of the Japanese Society for Cancer of the Colon and Rectum. Stomach and Intestine (Tokyo) 2011;46(4):442-448.

9. Tanahashi J, Daa T, Gamachi A, et al. Human HIS in Japan: its incidence, clinicopathologic features, and genotypic identification. Mod Pathol 2008;21(2):76-84.

10. Ushijima T, Sasako M. Focus on gastric cancer. Cancer Cell 2004;5(2):121-125.

11. Fukase K, Kato M, Kikuchi S, et al. Effect of eradication of Helicobacter pylori on incidence of metachrome gastric carcinoma after erndoscopic resection of early gastric cancer: an open-label, randomized controlled trial. Lancet 2008;372(9636):392-397. 


\section{Figure legends}

Figure 1 Endoscopic photograph showing SSA/P with synchronous adenocarcinoma

Figure 2 Photomicrograph showing SSA/P (HE staining)

Figure 3A Photomicrograph showing a HIS-positive "fringe formation" (anti-TP antibody staining)

Figure 3B Photomicrograph of HIS-positive tissue with no "fringe formation" showing the spirochetes within the crypts that tested HIS-positive in anti-TP antibody staining

Figure 4 Rates of HIS at each site in colon and rectum

Figure 5 Rates of HIS in SSA/Ps and controls

*Fisher's exact test 
Table 1 Comparison of SSA/P cases and controls

\begin{tabular}{lccc}
\hline & SSA/Ps & Controls & $P$ value \\
\hline Number of cases & 19 & 172 & \\
Number of lesions & 35 & 348 & $\mathrm{~ns}^{*}$ \\
Mean age (range) & $62.3(25-82)$ & $54.5(16-89)$ & $\mathrm{ns}^{* *}$ \\
Males/females & $12: 7$ & $115: 57$ & \\
\hline
\end{tabular}

*t-test

**Fisher's exact test; ns, not significant 



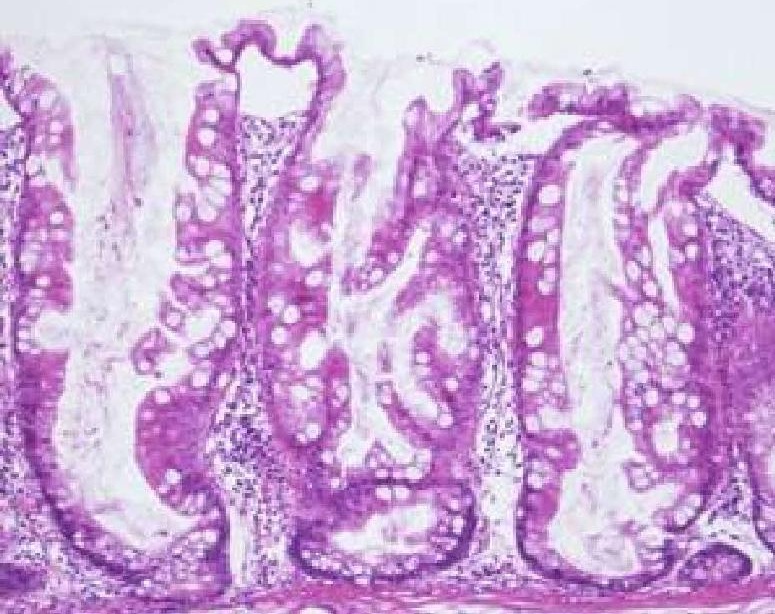




\section{T9.1\%}

I46 spectimens|

Aipht colon: $7.3 \%$

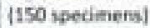

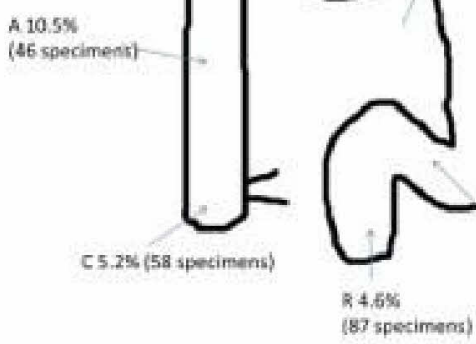

C5.2\% (58 specimens)
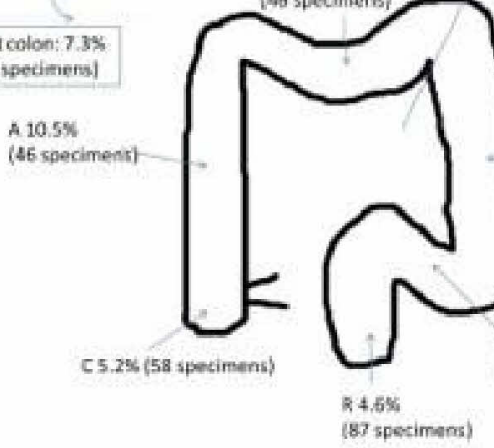

C. cecum, Ar accending colon, T: tranwerse colon, D. descendinc colon 5 : siemoid colon, R: rectum 


\section{PeDODLO1*}

ithen:

An:

IF:

Fल

부인

Her:

trix

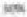

Wh

ini

E
Positine $52.6 \%$
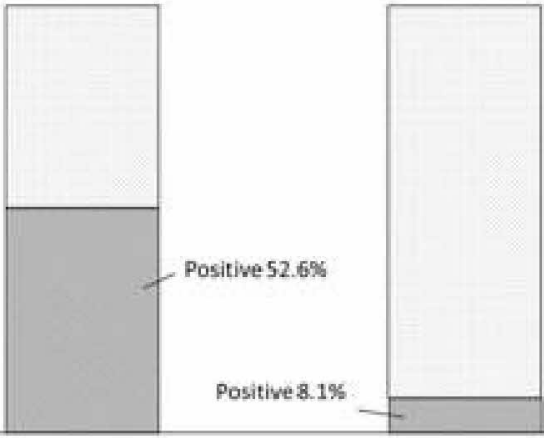

Control (n-172) 